\title{
Ultrasound and liver elastography - tools for detection and quantification of severity in nonalcoholic fatty liver disease (NAFLD) in the general population
}

\section{Ioan Sporea}

Department of Gastroenterology and Hepatology, "Victor Babeş" University of Medicine and Pharmacy, Timişoara, Romania

In the latter years in hepatology, due to new, very potent antiviral drugs that can eradicate or control viral replication in chronic hepatitis $\mathrm{C}$ and $\mathrm{B}$ patients, witnessed a change in focus has been witnessed: from chronic viral hepatitis to fatty liver disease. NAFLD (non-alcoholic fatty liver disease) affects approximately one quarter of the population in developed countries, while NASH (non-alcoholic steato-hepatitis) is present in approximatively $2-4 \%[1,2]$. Despite the fact that the course of NASH is quite long, the patients can develop liver cirrhosis and later hepatocellular carcinoma. In the last years, hepatologists have focused on how to find the patients at risk for NAFLD and NASH, how to predict their evolution and maybe to stratify the risk, considering that a huge cohort of asymptomatic subjects (millions of people in an area) are dealt with at any one time.

The answer to the first question - how to find them? is to evaluate the patients at risk for NAFLD and NASH: firstly the overweight and obese patients; secondly patients with type 2 diabetes: thirdly patients with metabolic syndrome and, of course, dyslipidemic subjects (many of them having all these risk factors) $[3,4]$. The second question is how to identify the subjects at risk to progress toward advanced liver disease. The general practitioner (GP) is the first gate of this strategy, being in direct contact with these patients, then the diabetologist who has most patients at risk under surveillance, usually for a long time (type 2 diabetes patients and dyslipidemic subjects), also the car-

Received Accepted

Med Ultrason

2017, Vol. 19, No 3, 247-249

Corresponding author: Prof. Ioan Sporea, MD, PhD

13, Snagov Street,

300482 Timisoara, Romania

Phone: +40 256309455

E-mail: isporea@umft.ro diologist who is following-up patients with metabolic syndrome. Finally, the hepatologist/gastroenterologist whose duty is to establish the disease severity, the prognosis and the best treatment approach for these patients.

The first step in screening the population at risk is to find liver steatosis; the second - to find if this fatty infiltration has a significant impact on the liver; and finally - for prognosis, to find if fibrosis is present.

The first aim - to discover steatosis and to estimate its severity - can be easily and inexpensively reached by using liver ultrasonography (US). No expensive ultrasound machines and no large ultrasound experience are required. The sensitivity of liver US for discovering and quantifying steatosis ranges between $60-80 \%$, even higher for severe steatosis [5], influenced by the physician's experience. In centers where a FibroScan device is available, a more objective evaluation of steatosis can be performed using CAP (Controlled Attenuation Parameter). For CAP, cut-off values were calculated for different degrees of steatosis, with accuracy ranging from 80 to $85 \%$ [6,7]. In patients undergoing CT or MRI for other purposes, both techniques can give valuable information concerning the severity of fatty infiltration of the liver, but none of them are screening tools.

Considering the availability, the low cost, and the relatively good sensitivity of US, it seems to be the best method to screen for steatosis in the general population. US can be performed by specialists or by a GP. The criticism for US can be that its accuracy is at its best only if fatty infiltration exceeds $20 \%$. But considering carefully the increased echogenicity of the liver with posterior attenuation and the liver/right kidney gradient, the method is quite sensitive, at least for experienced people [8].

The next question is how can inflammation, and especially fibrosis, be assessed in patients with risk factors 
for NAFLD. A surrogate for inflammation can be the level of aminotransferases (ASAT/ALAT ratio), useful for general practice and especially for GP's screening. We must advise the GP, the diabetologist and cardiologist, that any small increase of aminotransferases in risk subjects must be evaluated by the hepatologist. Other more sophisticated biological tests can be used, such as the NAFLD test or FibroMax. Some of them are expensive (FibroMax) and not widely available. Cytokeratin 18 was proposed as a surrogate test for inflammation detection and NASH [9].

But let's focus our discussion on liver fibrosis! This is the most important aspect, since it gives the prognosis. Liver fibrosis can be assessed by liver biopsy or non-invasively. But how can we speak about liver biopsy when NAFLD is an epidemic disease, with millions of cases? This method is too invasive for daily practice and can lead to complications [10].

What about liver elastography? Lately it has became a fashion! During ILC Amsterdam 2017, many papers and discussion were focused on this topic. More than 10 years ago, Transient Elastography (TE) arrived on the market, performed with a FibroScan device. It evaluates liver stiffness as a marker of fibrosis. Many papers demonstrated its good value of initially in chronic viral hepatitis and later also in NAFLD [11]. The new XL probe (for obese subjects), that completed the $\mathrm{M}$ probe, improved the method's feasibility to more than $90 \%$ [12]. Thus, the vast majority of patients can be evaluated for fibrosis severity by TE. Cut-off values for various stages of fibrosis were calculated for different etiologies, so that TE became a "a must to have" in clinical hepatological practice.

During the last 5 years, other US based elastographic techniques were developed: point shear wave elastography (pSWE) or real time elastography (2D-SWE), all integrated into ultrasound machines, can provide liver steatosis and liver stiffness evaluation in the same session. Very recently, an update on the EFSUMB Guidelines and recommendations on liver elastography have been published [13]. We expect that in the next few years, ultrasound systems with stiffness evaluation will be common in daily practice. The cost of such a system is still high (despite the fact that some companies have introduced the elastographic module in their mid-class ultrasound machines), but many hepatologists (and maybe GPs) would like to have such a system to evaluate a pathology that is increasing annually. The maintenance cost for such a system is very low and thus it can be used for a long time, without supplementary costs. One of the companies released very recently an ultrasound system able to perform both 2D-SWE and TE. Another company tried to combine pSWE and 2D-SWE in the same ultrasound machine.

Thus, very soon, the hepatologist (radiologist, diabetologist, maybe the cardiologist, or GP) will be able to start screening people at risk, in order to find significant liver steatosis and significant liver fibrosis using ultrasound machines and liver elastography. In some areas, such as the USA, where a lot of energy has been invested in Magnetic Resonance Elastography (MRE), this method can be a competitor. But it is still very expensive and probably not suitable for screening purposes. The competition will be between the FibroScan with CAP (that quantifies steatosis and fibrosis severity) and ultrasound machines with elastography modules. Probably the analysis of the acquisition and maintenance costs will be the decisive factor. Of course, there is a place also for biological tests, but maybe decreasing the cost for complex tests (such as FibroMax) should be recommended.

In centers where systems are able to evaluate the severity of liver steatosis and liver fibrosis, I believe that it is the moment to start screening for the presence of fatty liver and fibrosis in patients with risk factors (type 2 diabetes, obese, dyslipidemia, metabolic syndrome) [14]. Early diagnosis of significant disease is the best moment to start therapy (life style changes or drug therapy). NAFLD is the more prevalent disease in hepatology now, in the developed world, and now is the time to start the fight against this disease!

\section{References}

1. Adams LA, Angulo P, Lindor KD. Nonalcoholic fatty liver disease. CMAJ 2005;172:899-905.

2. Lazo M, Hernaez R, Eberhardt MS, et al. Prevalence of nonalcoholic fatty liver disease in the United States: the Third National Health and Nutrition Examination Survey, 1988-1994. Am J Epidemiol 2013;178:38-45.

3. Harris R, Harman DJ, Card TR, Aithal GP, Guha IN. Prevalence of clinically significant liver disease within the general population, as defined by non-invasive markers of liver fibrosis: a systematic review. Lancet Gastroenterol Hepatol 2017;2:288-297.

4. Koehler EM, Plompen EP, Schouten JN, et al. Presence of diabetes mellitus and steatosis is associated with liver stiffness in a general population: The Rotterdam study. Hepatology 2016;63:138-147.

5. Palmentieri B, de Sio I, La Mura V, et al. The role of bright liver echo pattern on ultrasound B-mode examination in the diagnosis of liver steatosis. Dig Liver Dis 2006;38:485-489.

6. Shi KQ, Tang JZ, Zhu XL, et al. Controlled attenuation parameter for the detection of steatosis severity in chronic liver disease: a meta-analysis of diagnostic accuracy. J Gastroenterol Hepatol 2014;29:1149-1158. 
7. Karlas T, Petroff D, Sasso M, et al. Individual patient data meta-analysis of controlled attenuation parameter (CAP) technology for assessing steatosis. J Hepatol 2017;66:10221030.

8. Kwon HJ, Kim KW, Jung JH, et al. Noninvasive quantitative estimation of hepatic steatosis by ultrasound: a comparison of the hepato-renal index and ultrasound attenuation index. Med Ultrason 2016;18:431-437.

9. Castera L. Noninvasive Evaluation of Nonalcoholic Fatty Liver Disease. Semin Liver Dis 2015;35:291-303.

10. Poynard T, Ratziu V, Bedossa P. Appropriateness of liver biopsy. Can J Gastroenterol 2000;14:543-548.

11. Kwok R, Tse YK, Wong GL, et al. Systematic review with meta-analysis: non-invasive assessment of non-alcoholic fatty liver disease - the role of transient elastography and plasma cytokeratin-18 fragments. Aliment Pharmacol Ther 2014;39:254-269.

12. Sporea I, Şirli R, Mare R, Popescu A, Ivașcu SC. Feasibility of Transient Elastography with M and XL probes in real life. Med Ultrason 2016;18:7-10.

13. Dietrich CF, Bamber J, Berzigotti A, et al. EFSUMB Guidelines and Recommendations on the Clinical Use of Liver Ultrasound Elastography, Update 2017 (Long Version). Ultraschall Med 2017 Apr 13. doi:10.1055/s-0043-103952.

14. Mare R, Sporea I, Sirli R, et al. Transient Elastography with Controlled Attenuation Parameter (CAP) - a tool for liver disease screening in type 2 diabetes mellitus patients. J Gastrointestin Liver Dis 2017;26 (Suppl 2):31. 